\title{
A simple interaction law for viscous-inviscid interaction
}

\author{
Arthur E. P. Veldman
}

Received: 25 May 2009 / Accepted: 30 July 2009 / Published online: 14 August 2009

(C) The Author(s) 2009. This article is published with open access at Springerlink.com

\begin{abstract}
The viscous-inviscid interaction (VII) philosophy for modelling aerodynamic boundary layers is discussed. 'Traditionally' the shear-layer equations are solved with pressure prescribed by the inviscid flow, but then the solution breaks down in a singularity related to flow separation. In the quasi-simultaneous coupling approach this singularity is overcome by making use of an interaction law. A novel mathematical analysis is presented of the essential properties of such interaction laws, which is based on classical theory for non-negative matrices. The performance of a highly simplified interaction law is demonstrated for separated airfoil flow beyond maximum lift.
\end{abstract}

Keywords Boundary-layer flow · Flow separation · Goldstein singularity · Matrix analysis · Quasi-simultaneous coupling

\section{Introduction}

The accurate and fast prediction of viscous flow over two- and three-dimensional surfaces is an important problem in aerodynamics. The continuing advances in efficiency and accuracy of numerical algorithms, together with the increasing speed and memory size of computers, are enabling viscous flows to be calculated by methods that solve the full (Reynolds-averaged) Navier-Stokes equations. Nevertheless, whilst Navier-Stokes simulation potentially offers generality, the computational requirements still limit their use for practical application, especially within a design optimization environment where a large number of configurations is to be considered.

A possible alternative for the prediction of viscous flow over airfoil sections and wings, is to use the 'older' technique of viscous-inviscid interaction (VII). Here, an inviscid-flow solver is coupled to a viscous boundarylayer calculation method to provide a composite, approximate solution of the Navier-Stokes equations. For many cases of aerodynamic interest the coupled solution matches experimental data as well as Navier-Stokes simulation, and this at much lower computational cost; see e.g. [1,2].

VII methods 'owe' their existence to Prandtl's identification [3] of a thin shear layer close to a solid surface, where viscous forces are dominantly present: the boundary layer. In aerodynamic applications (Fig. 1), the boundary

A. E. P. Veldman $(\varangle)$

Institute of Mathematics and Computing Science, University of Groningen, P. O. Box 407, 9700 AK Groningen, The Netherlands

e-mail: a.e.p.veldman@rug.nl; veldman@math.rug.nl 
Fig. 1 Subdivision of the flow field around an airfoil in an inviscid flow region and a viscous shear layer (exaggerated in thickness)

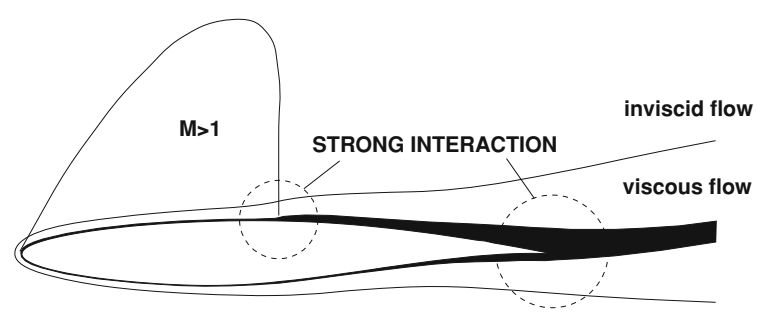

layer is driven by the inviscid pressure distribution $p_{e}$ or equivalently, because of Bernoulli's law, by its related streamwise velocity $u_{e}$. In the boundary layer the streamwise velocity component is reduced to zero in order to comply with the no-slip condition at the surface, and the body appears thicker (and smoother) than its original geometry. The resulting effective shape is called the displacement body. It is obtained by moving the original body surface outward over a distance $\delta^{*}$, the displacement thickness.

In (most) situations of attached flow, the boundary layer provides only a small perturbation to the outer inviscid flow. Together with the above way of reasoning, this strongly suggests to first calculate the inviscid flow as if there were no boundary layer. The resulting pressure distribution then is fed into the shear-layer equations to obtain its displacement effect. Herewith a correction to the outer flow can be computed. If desired, this process, called the direct method, can be repeated until (hopefully) convergence.

However, when regions of reversed flow are present, the direct boundary-layer calculation breaks down. In 1948, Goldstein [4] presented an extensive study of the breakdown process; since then the singularity at flow separation bears his name. He came up with a number of possible causes; however, at that time no definitive conclusion could be given.

A few years later, in 1953, Lighthill [5] shed his light on the related issue of upstream influence in subsonic and supersonic flow (see also [6]). For supersonic flow, it took only a few years longer to grasp the nature of the interaction near a point of flow separation. In 1959, Hayes and Probstein wrote in their monograph on hypersonic flow [7, p. 365]:

$\ldots$ in general it requires solving simultaneously the integrated momentum and energy equations and the inviscid flow relation describing the pressure along the curve $y=\delta^{*}(x)$.

For subsonic flow, it was not until 1966 that a firm indication on how to prevent Goldstein's singularity was given, when Catherall and Mangler [8] presented calculations of a boundary layer with prescribed $\delta^{*}$. At about the same time, a further clue was provided by the asymptotic triple-deck theory introduced by Stewartson [9], Neiland [10] and Messiter [11]. Again, “... a definite loss of hierarchy ..." between the inviscid flow region and the boundary layer was indicated, to quote Lagerstrom in 1975 [12, p. 209].

This lack of hierarchy should also be visible in the numerical information exchange between boundary layer and inviscid flow, thus guiding its appropriate numerical iterative treatment. In this spirit, in the late 1970s alternatives for the direct VII method were presented. An obvious choice is to reverse the order of information exchange. This inverse method survives in flow separation, but its convergence is extremely slow. Henceforth, LeBalleur [13] and Carter [14] mixed both methods into the semi-inverse method, where both flow regions are solved with prescribed $\delta^{*}$, which is then updated based on the difference in the respective $u_{e}$ distributions.

Another idea, in the spirit of Lagerstrom [12], was to avoid any hierarchy in the treatment of both flow regions by solving the viscous and inviscid flow equations simultaneously $[15,16]$. However, a fully simultaneous approach would require both sets of flow equations to be merged into one big system, which is quite complicated in software terms, and defies any flexibility in flow modeling. Hence, an attempt was made to approximate such a simultaneous approach, without complicating the (often existing) software. Thus the concept of the interaction law was introduced: a simple, yet powerful, description of the inviscid flow, which can easily be solved simultaneously with the boundary-layer equations $[17,18]$. Several examples of interactive calculations have been presented in the literature 
[19-21]. However, the used interaction laws were still rather complex, motivated by the desire to keep their physical contents as close as possible to the real physics.

In the present paper we will extend our point of view and analyze the quasi-simultaneous approach in a mathematical way. Subsequently, the interaction law will be simplified such that only its bare essentials remain. We end up with a method which is very close to the classical direct method, and yet has no problems with reversed flow. This is demonstrated with a calculation of airfoil flow beyond maximum lift.

\section{The flow model}

\subsection{Shear layer}

The above situation will be discussed from a specific flow model for the shear layer, namely an integral formulation. In this way, the origin of the singularity at separation can be pointed out explicitly. Nevertheless, this specific choice does not sacrifice the generality of the considerations and conclusions. The alternative field description of boundary layers, see e.g. [22, Chap. 12], possesses the same behaviour, albeit less tractable for theoretical analysis. The discussion is held in two dimensions, where the situation is more extreme than in three dimensions; see e.g. [23].

In most VII methods, the flow is described by the Von Kármán's integral boundary-layer equation, presented already in 1922 [24]. In unsteady formulation, it can be written as

$$
\frac{1}{u_{e}^{2}} \frac{\partial}{\partial t}\left(u_{e} \theta H\right)+\frac{\partial \theta}{\partial x}+\frac{\theta}{u_{e}}(2+H) \frac{\partial u_{e}}{\partial x}=\frac{1}{2} c_{f},
$$

where $\theta$ represents the momentum thickness, $H$ the shape factor $\left(H \equiv \delta^{*} / \theta\right)$, and $c_{f}$ the coefficient of skin friction. This equation is combined with a number of experimentally obtained algebraic relations between several of the appearing variables. Various proposals have been made in the literature; for an overview see e.g. [25].

Here, we will apply the unsteady version of Head's entrainment method [26], in which the entrainment coefficient $C_{E}$ of the shear layer is defined in terms of Head's shape factor $H_{1} \equiv\left(\delta-\delta^{*}\right) / \theta$ (with $\delta$ the shear-layer thickness) through

$$
\frac{1}{u_{e}} \frac{\partial}{\partial t}\left(\theta\left(H_{1}+H\right)\right)+\frac{1}{u_{e}} \frac{\partial}{\partial x}\left(u_{e} \theta H_{1}\right)=C_{E} .
$$

Head's idea is that the entrainment coefficient $C_{E}$ only depends on $H_{1}$. Experimental data suggest the following relation

$C_{E}=0.0306\left(H_{1}-3.0\right)^{-0.6169}$.

The most important closure relation expresses $H_{1}$ in terms of $H$ only. Figure 2 summarizes versions of this relation as used at ONERA [27], RAE [28] and NLR [29]. All relationships show a minimum at or near the onset of flow separation where $H \approx 2.7$. This minimum turns out to play an essential role in the interaction process, as we will see below. For larger values of $H$ the curves disagree, but experimental data to support these curves was (and is still) rare. Figure 1 of Lock and Williams' review paper [25] summarizes the existing data. In the calculations to follow, we have made use of Houwink and Veldman's formulation [29]

$H \leq 4: H_{1}=\frac{1}{2} \frac{h_{t}\left(h_{t}+2\right)}{h_{t}-1}, \quad H>4: H_{1}=1.75+\frac{5.52273 h_{t}}{h_{t}+5.818181}$,

where $h_{t}=\min \left\{H, \frac{1}{2}(H-2.732)+2.732\right\}$. For large values of $H$, this relation has a smaller slope than the other two, which turns out to be beneficial, as we will see in Sect. 6.

Finally, a closure relation for the skin friction $c_{f}$ is required. The 'classical' relationship is provided by Ludwig and Tilmann, but we will use Green's modification as it can provide negative values for $c_{f}$. It is a function of the shape factor $H$ and the Reynolds number $\operatorname{Re}_{\theta}$ based on the momentum thickness [29-31]:

$c_{f}=c_{f_{0}}\left(\frac{0.9}{H h_{0}-0.4}-0.5\right)$, 
Fig. 2 Some $H-H_{1}$ relationships as used at ONERA [27], RAE [28] and NLR [29] around 1980. The three relations agree on having a minimum near $H \approx 2.7$, corresponding with the onset of separation. For larger values of $H$ the curves disagree, but experimental data to support these curves was rare at that time

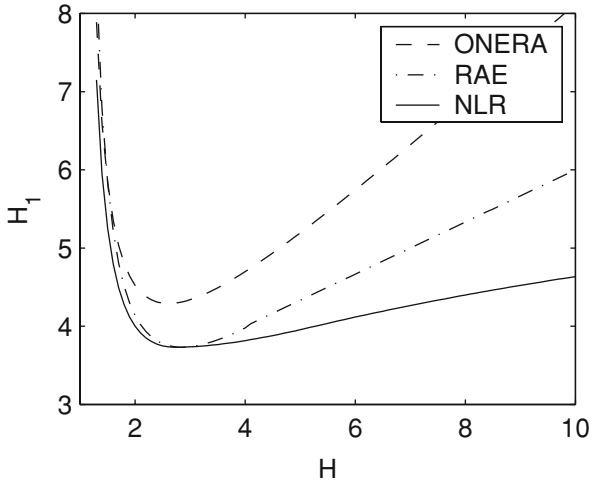

where

$c_{f_{0}}=\frac{0.01013}{\log _{10} \operatorname{Re}_{\theta}-1.02}-0.00075$ and $h_{0}=1.0-6.55 \sqrt{\frac{1}{2} c_{f_{0}}}$.

It is our experience that the influence of this modification on the computational results is very modest, apart from the 'visibility' of the separated flow region through the sign of $c_{f}$.

Equations $1-5$ form the viscous flow model. As soon as the external velocity $u_{e}$ is known, in principle sufficient equations are available to find their solution. However, as indicated before, not always a solution can be found; more on this issue follows below.

The two differential equations (1) and (2) can be combined into a $2 \times 2$-system for the unknowns $\theta$ and $H$ (rather than $H_{1}$ )

$A \frac{\partial \phi}{\partial t}+B \frac{\partial \phi}{\partial x}=R, \quad \phi=\left(\begin{array}{c}\theta \\ H\end{array}\right)$ and $R=\left(\begin{array}{c}\frac{1}{2} c_{f}-\frac{\theta}{u_{e}}(2+H) \frac{\partial u_{e}}{\partial x}-\frac{H \theta}{u_{e}^{2}} \frac{\partial u_{e}}{\partial t} \\ C_{E}-\frac{H_{1} \theta}{u_{e}} \frac{\partial u_{e}}{\partial x}\end{array}\right)$.

The coefficient matrices $A$ and $B$ are given by

$A=\frac{1}{u_{e}}\left(\begin{array}{cc}H & \theta \\ H_{1}+H & \theta\left(\frac{\mathrm{d} H_{1}}{\mathrm{~d} H}+1\right)\end{array}\right)$ and $B=\left(\begin{array}{cc}1 & 0 \\ H_{1} & \theta \frac{\mathrm{d} H_{1}}{\mathrm{~d} H}\end{array}\right)$.

\subsection{Coupling with inviscid flow}

The influence of the shear layer on the external flow is described in terms of its displacement thickness $\delta^{*}$. In 1958, Lighthill [32] presented several interpretations of the displacement thickness and of the way in which it influences the outer inviscid flow. In the present paper we prefer to apply the surface transpiration concept, where at the original position of the body geometry a non-zero normal velocity is prescribed

$v=\frac{\mathrm{d}}{\mathrm{d} x}\left(u_{e} \delta^{*}\right)$.

This transpiration velocity acts as a boundary condition for the inviscid-flow solver. To understand the physical information exchange between both flow regions, it is not necessary here to use a sophisticated inviscid-flow model. During the more detailed mathematical analysis of the viscous-inviscid problem as presented here, a simple thin-airfoil approximation of the external inviscid flow turns out sufficiently useful:

$u_{e}(x)=u_{e_{0}}(x)+\frac{1}{\pi} \int_{\Gamma} \frac{\mathrm{d}}{\mathrm{d} \xi}\left(u_{e} \delta^{*}\right) \frac{\mathrm{d} \xi}{x-\xi}$, 
where $u_{e_{0}}$ is the edge velocity without displacement effects. If convenient, and when accuracy is not of first concern, the factor $u_{e}$ under the integral can be simplified further to $u_{e_{0}}$ or even $U_{\infty}$ (the free-stream velocity).

For completeness, at the end points of the considered flow domain boundary conditions for $\delta^{*}$ are required. At the upstream boundary the condition follows from the oncoming flow profile; at the downstream boundary one may simply use $\mathrm{d} \delta^{*} / \mathrm{d} x=0$.

\section{Steady and unsteady singularities}

\subsection{Steady flow without interaction}

As soon as the flow wants to separate from the surface, the above strategy breaks down; in particular, no solution of the boundary-layer equations can no longer be found. In a now famous paper from 1948, Goldstein [4, p. 45] describes unpublished attempts by Hartree to obtain numerical solutions of the boundary-layer equations near separation:

All computations in which any attempt was made to obtain real accuracy at and near separation seem to have met with considerable difficulty. As a result of his computations, Professor Hartree was convinced that there was a singularity in the solution at the position of separation.

Goldstein "undertook to try to find some formulae that would hold near this singularity and would help in finishing the computation." He investigated several possible reasons for this failure near separation. One of his suggestions is given on p. 50 of his paper [4]:

Another possibility is that a singularity will always occur except for certain special pressure variations in the neighbourhood of separation, and that, experimentally, whatever we may do, the pressure variations near separation will always be such that no singularity will occur.

Inspired by Goldstein's research, since then a number of important steps forward have been made, as described in the Introduction.

A confirmation of these ideas in an engineering context came thirty years later, when LeBalleur [13,27] presented his vision on the $H-H_{1}$ relation discussed in Sect. 2. With reference to the formulation (6), the steady viscous-flow problem can be written as

$B \frac{\partial \phi}{\partial x}=R$,

with $B$ given in (7) and $R$ in (6). From (7) it is immediately clear that the matrix $B$ is singular as soon as $\mathrm{d} H_{1} / \mathrm{d} H=0$, after which the calculations come to an (untimely) end. In less mathematical terms, the reason for the breakdown at separation is the minimum in Fig. 2: not for every $H_{1}$ a value for $H$ can be obtained. Goldstein's quoted suggestion turns out correct!

With this hindsight, it becomes clear that the boundary layer itself wants to determine the pressure gradient near separation, instead of having it prescribed through the external flow. The interaction hierarchy between inviscid flow and boundary layer is changing from weak interaction (where the inviscid flow region dominates) into strong interaction (where the boundary layer has an equal say); cf. [24, Chap. 14].

\subsection{Steady flow with interaction}

When interaction is included, $u_{e}$ should be considered an unknown, together with $\theta$ and $H$. The interaction law forms the additional equation that is required to facilitate the additional unknown. Thus, the derivatives of $u_{e}$, which without interaction are 'hidden' in $R$, have to be made visible and shifted to the left-hand side of (6). A convenient way is to eliminate $u_{e}$ immediately from the interaction law. When assumed linear, an interaction law looks like 
$u_{e}-c \delta^{*}=R H S$, where the details of the right-hand side are not relevant here. The coefficient in front of $\delta^{*}$ is going to be positive, as we will see later on, i.e., $c>0$.

Using the interaction law, we can transfer the derivatives of $u_{e}$ in the right-hand side of (6) into derivatives of $\delta^{*}$, and ultimately in those of $\theta$ and $H$. In the end, the steady system of equations becomes

$\hat{B} \frac{\partial \phi}{\partial x}=\hat{R}$ with $\hat{B}=\left(\begin{array}{cc}1+C H(2+H) & C \theta(2+H) \\ H_{1}(1+C H) & \theta \frac{\mathrm{d} H_{1}}{\mathrm{~d} H}+C \theta H_{1}\end{array}\right)$, where $C=\frac{c \theta}{u_{e}}>0$.

Again, the right-hand side $\hat{R}$ is not important here.

The relevant question is "What happens with Goldstein's singularity?" The answer follows from the singularities of the matrix $\hat{B}$. A little analysis shows that it becomes singular when

$\frac{\mathrm{d} H_{1}}{\mathrm{~d} H}=\frac{C H_{1}(1+H)}{1+C H(2+H)}$.

As the right-hand side in (10) is positive (note that $H, H_{1}$ and $C$ are all positive in practical situations), and looking at the shape of the $H-H_{1}$ curve, it is found that Goldstein's singularity moves to larger values of $H$. If $C$ is large, and depending on the asymptotic shape of the $H-H_{1}$ relation, it might even disappear towards infinity. Whether this has a physical meaning is unclear at the moment. It would require more knowledge on physically realistic $H-H_{1}$ relations deep in the separated flow regime (where hardly any experimental data are available).

\subsection{Unsteady flow}

In the unsteady case, the matrix $A$ in the system (6) plays an important role. The system is hyperbolic, with its characteristic directions $\lambda$ determined by $|\lambda A-B|=0$, where $A$ and $B$ are given in (7). Some analysis reveals that for attached flow both characteristic directions are positive. When $B$ becomes singular, i.e., at the minimum of $H_{1}$, one of the directions changes sign. This physically corresponds to information flowing in the upstream direction, due to the presence of reversed flow. Cousteix and Houdeville, see [33], have analyzed this situation extensively. In unsteady calculations this change of direction should be acknowledged to obtain a stable scheme.

However, when $A$ becomes singular one characteristic direction blows up and the whole unsteady system degenerates. By analyzing $A$ 's determinant, see (7), this happens when

$$
\frac{\mathrm{d} H_{1}}{\mathrm{~d} H}=\frac{H_{1}}{H} \text {. }
$$

It is conjectured that this breakdown corresponds with the Van Dommelen-Shen singularity for unsteady flow [34]. Further research into this presumed relation is currently in progress. Addition of interaction does not help, as also interactive unsteady calculations come to an end when the extent of separation increases [35]. It would be very interesting to try to relate this breakdown to the asymptotic theory of marginal separation [36-38].

\subsection{Bird's eye view}

The above shows that all relevant information, including the generation of singularities, is gathered in the chosen $H-H_{1}$ relation. The interesting points are its minimum and the (eventual) point where the tangent passes through the origin; Fig. 3 (left) summarizes the situation.

The situation can also be explained in terms of the local relation between $u_{e}$ and $\delta^{*}$; for convenience we restrict ourselves to the steady situation. When looking at a fixed boundary-layer station $x_{i}$, the relation can be pursued between $u_{e}\left(x_{i}\right)$ and $\delta^{*}\left(x_{i}\right)$. It is found [39,40] to have a similar shape as the $H-H_{1}$ relation, again with a minimum at the point of flow separation. A sketch of this relation is given in Fig. 3 (right). Obviously, prescription of $u_{e}$ may run into problems. But also a way out of the problem can be seen. The boundary-layer relation in the graph is 

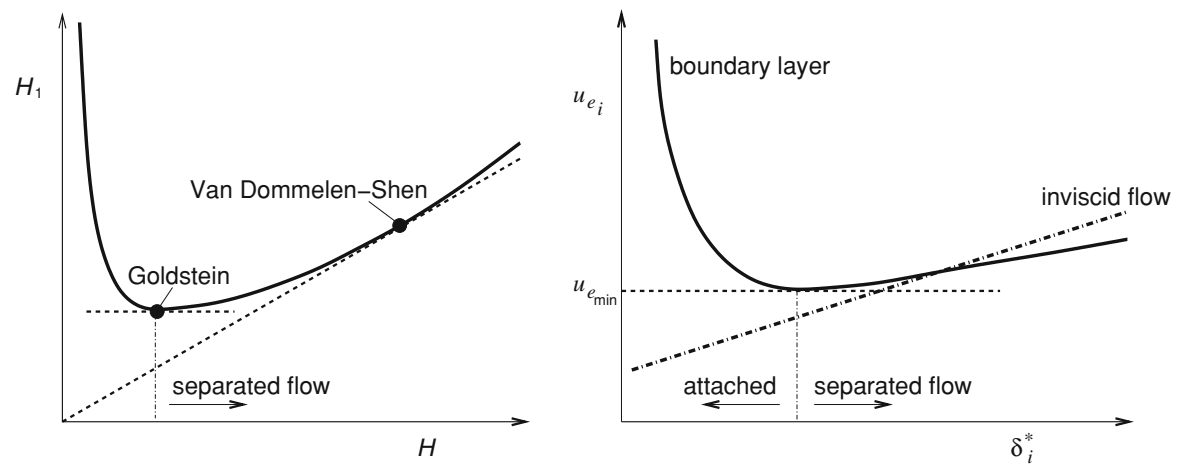

Fig. 3 Left Singular points related to the $H-H_{1}$ relation. Right Boundary layer and inviscid flow combined in terms of $u_{e}$ and $\delta^{*}$. Not for every slope of the inviscid flow relation a solution can be obtained

to be combined with an inviscid flow relation. When the latter, supposed linear for convenience, has a sufficiently positive slope-recall we announced to have $c>0$ in (9) - a solution to the combined viscous-inviscid problem appears possible! In the discussion to follow this graph will play an essential role.

\section{Quasi-simultaneous VII and the interaction law}

We will enlarge the abstraction of the flow model from Sect. 2, by focussing on the streamwise velocity $u_{e}$ and the displacement thickness $\delta^{*}$ (thus effectively eliminating $H$; as the equations are complicated enough already, we will not do this explicitly). Both flow domains provide a relation between these two quantities, i.e., in principle we have two equations with two unknowns. In this abstract setting, the steady version of the coupled VII problem can be written as

$\left.\begin{array}{l}\text { external inviscid flow: } u_{e}=E \delta^{*} \\ \text { boundary-layer flow: } u_{e}=V \delta^{*}\end{array}\right\} \Rightarrow(E-V) \delta^{*}=0$.

The quasi-simultaneous method tries to work around Goldstein's singularity $-V$ is singular at separation and re-attachment—by avoiding any iterative hierarchy. The basic idea is to inform the boundary layer instantaneously how the external flow will react on changes inside the boundary layer. Hereto, a sufficiently accurate, yet simple, approximation of the external inviscid flow is introduced, denoted as $u_{e}=I \delta^{*}$. This interaction law is to be solved simultaneously with the boundary-layer equations, i.e.,

$\left.\begin{array}{l}u_{e}^{(n)}-I \delta^{*(n)}=(E-I) \delta^{*(n-1)} \\ u_{e}^{(n)}-V \delta^{*(n)}=0\end{array}\right\} \Rightarrow(I-V) \delta^{*(n)}=(I-E) \delta^{*(n-1)}$,

where $(n)$ counts the VII iterations.

The first task of the interaction law is to make the numerical calculations survive, with Goldstein's singularity as the ultimate challenge. Here a good description of the local interactive physics is essential. Once the calculations keep running, as a second task a swift iterative convergence towards the viscous-inviscid solution of (12) can be pursued. The VII iterations (13) have to account for the difference between the external flow $E$ and its approximation $I$; also here an interaction law that possesses essential properties of the 'exact' inviscid flow is preferable. However, it is stressed that the choice of the interaction law does not influence the finally converged solution of (13). The latter is solely determined by the choices made for the inviscid and viscous operators $E$ and $V$ in (12). The interaction law only controls the 'ease' with which the viscous-inviscid solution is obtained.

Thus, the final question arises, namely how to choose the interaction law. As indicated, here the physics of the problem plays a central role. A fair description of how an inviscid flow reacts on displacement effects is delivered by thin-airfoil theory, in a simple form given by (8). Also triple-deck theory provides this type of approximation [41], 
which makes (8) a good candidate as an interaction law. In fact, this interaction law (describing thickness effects) together with its anti-symmetric counterpart (describing camber effects) has been used successfully in subsonic as well as transonic airfoil/wake calculations [19,21].

The implementation of an interaction law, be it the thin-airfoil expressions for thickness and camber or an influence matrix of a panel method, can be cumbersome. Therefore, it is worthwhile to investigate how much the interaction law can be simplified without being struck by Goldstein's singularity. The viscous-inviscid convergence is likely to deteriorate, but the effort to adapt an existing 'traditional' boundary-layer code to separated flow computations will be smaller. Thus, referring to the quasi-simultaneous formulation (13), the question is

How 'simple' can the interaction law $I$ be chosen?

This question has been investigated in the $\mathrm{PhD}$ thesis of Coenen [42], prepared under the author's supervision; see also [43]. She has performed a mathematical analysis based on the theory for non-negative matrices [44, Chap. 2] and the closely related $M$-matrices [45, Chap. 2.7; 46, Chap. 2.5]. We will first present the theory as developed by Coenen. Thereafter, the usefulness of the theory will be demonstrated on a realistic flow problem.

\section{A model problem}

To shape the theory, in the spirit of Carter and Wornom [47] the flow past an indented plate has been selected as a model problem (Fig. 4). The dent is about one unit wide and chosen quite deep in comparison with the boundary-layer thickness. Note that we do not intend to produce a physically accurate solution of this flow problem. The model problem is only meant to be a challenging and representative numerical test case for a VII algorithm.

The dent is placed in a uniform flow with unit velocity $U_{\infty}$. The Reynolds number based on unit length is $10^{8}$. The boundary layer is modelled with the steady version of Head's entrainment method (2). The external inviscid flow is described by the thin-airfoil expression (8). It is our aim to construct even simpler interaction laws for this case. Let us first collect some properties of the corresponding discrete operators $E$ and $V$.

\subsection{External flow}

The integral (8), in its simplest form with $U_{\infty}$ replacing $u_{e}$ in the integrand, is discretized on a uniform grid with mesh size $h$. The displacement thickness $\delta^{*}$ is interpolated by a piece-wise linear function; only on the two intervals adjacent to the Cauchy principal value a quadratic interpolation is used:

$$
\begin{aligned}
E\left[\delta^{*}\right]\left(x_{i}\right)-u_{e 0}\left(x_{i}\right) & \equiv \frac{U_{\infty}}{\pi} \int_{\Gamma} \frac{\mathrm{d} \delta^{*}}{\mathrm{~d} \xi} \frac{\mathrm{d} \xi}{x_{i}-\xi}=\frac{U_{\infty}}{\pi}\left\{\int_{x_{i-1}}^{x_{i+1}}+\sum_{j \neq i-1, i} \frac{1}{\pi} \int_{x_{j}}^{x_{j+1}}\right\} \frac{\mathrm{d} \delta^{*}}{\mathrm{~d} \xi} \frac{\mathrm{d} \xi}{x_{i}-\xi} \\
& \approx-\left.\frac{2 h U_{\infty}}{\pi} \frac{\mathrm{d}^{2} \delta^{*}}{\mathrm{~d} \xi^{2}}\right|_{i}+\left.\frac{h U_{\infty}}{\pi} \sum_{j \neq i-1, i} \frac{\mathrm{d} \delta^{*}}{\mathrm{~d} \xi}\right|_{j+1 / 2} \log \left|\frac{i-j}{i-j-1}\right| .
\end{aligned}
$$

Fig. 4 Geometry sketch of indented plate geometry

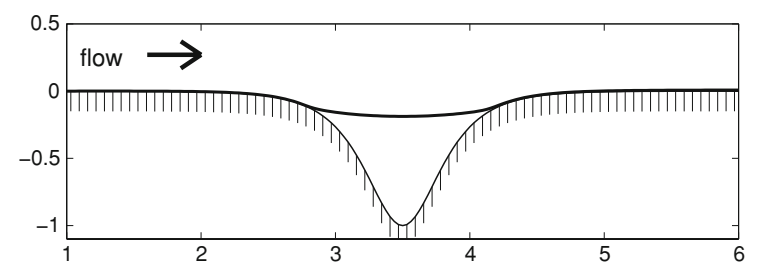


After discretization of the $\xi$-derivatives in (14), the corresponding discrete matrix $E$ is symmetric, positive definite with diagonal $4 U_{\infty} / \pi h$, and with non-positive off-diagonal entries; hence $\mathrm{E}$ is a symmetric, positive definite $M$-matrix (see Theorem A.3 in Appendix).

\subsection{Boundary-layer flow}

Referring e.g. to [39,40], we observe that a discrete boundary-layer operator $V$ typically is lower-diagonal, with negative diagonal entries in boundary-layer stations with attached flow and (slightly) positive diagonal entries in station with reversed flow. This can be seen from the slope of the $\delta^{*}-u_{e}$-relation in Fig. 3, which is positive for attached flow and negative for separated flow. For attached flow this implies that the diagonal of $E-V$ is strengthened. However, in separated flow regions its diagonal is weakened, which opens room for eigenvalues to move into the unstable half plane. Physically this is related to the flow becoming unsteady.

\section{Matrix theory of viscous-inviscid interaction}

To develop the theory, we will interpret the continuous operators in the quasi-simultaneous method (13) as discrete matrices without changing the notation. Thus the discrete equation

$(E-V) \delta^{*}=0$

is solved, using an iterative splitting

$(I-V) \delta^{*(n)}=(I-E) \delta^{*(n-1)}$.

We will analyze this iterative approach to solve (15) by theoretical means. As we are considering situations with steady flow, it is natural to make the following assumption; moreover, it allows theory to be developed.

Assumption 1 The matrices $E-V$ and $I-V$ are assumed to be irreducible, (positive) stable $M$-matrices, i.e., they have positive diagonal entries and non-positive off-diagonal entries, whereas all of their eigenvalues lie in the stable positive half plane (and the matrices cannot become singular).

In general terms, the convergence rate of the viscous-inviscid iterations (16) improves the more $I$ resembles $E$. However, with more complicated $I$ the solution of the shear-layer equations plus interaction law will slow down. We will present theorems on how the choice of $I$ influences these two iteration processes separately. The performance of their combination can only be found from experimenting with the model problem. Another relevant item for practical calculations is the robustness of the iterations. Because the problem is highly nonlinear, one has to be careful in choosing the iterative path that should lead towards the converged solution. Also here the influence of the choice of $I$ can be analyzed theoretically.

\subsection{Viscous-inviscid iterations}

Obviously, when $I=E$ no VII iterations are necessary. However, we would like to simplify $I$. But how? The physical idea for constructing an interaction law is that it should contain the essential part of the interaction with the external flow, i.e., it should contain the local effect of the interaction. From a mathematical, iterative point of view, diagonal matrices are very tractable. Hence, physical and mathematical ideas come together. Both suggest to define interaction laws in which some outer off-diagonals of $E$ are omitted, or in which its diagonal is enhanced (Fig. 5). With $E$ having non-positive off-diagonal entries, this results in a class of matrices for which $I \geq E$. The next theorem guarantees that, indeed, such a choice leads to convergent VII iterations.

Theorem 1 Let the interaction law I be constructed such that I $\geq$ E. Moreover, let E and I satisfy Assumption 1. Then: 

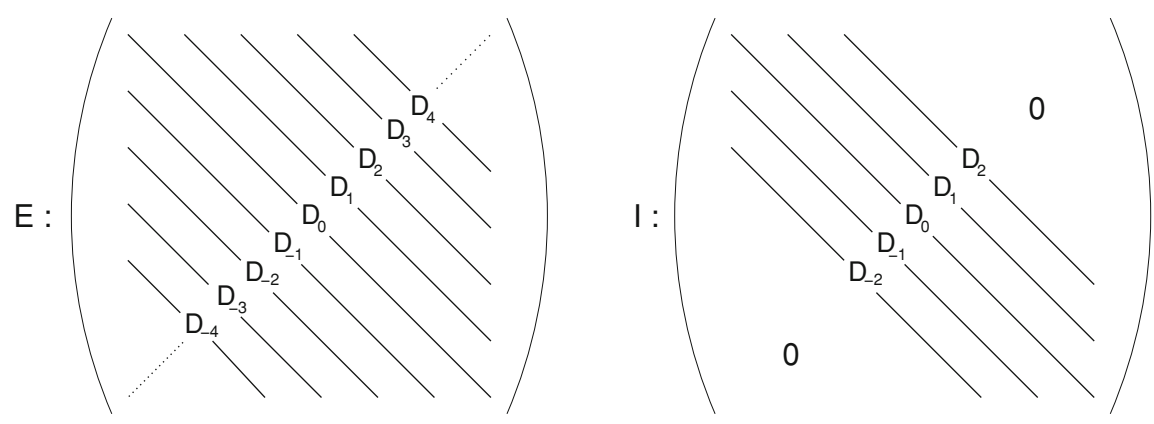

Fig. 5 The interaction law I (right) may be obtained from the external flow matrix $E$ (left) by omitting some off-diagonals (here only two diagonals on each side have been retained)

(1) The viscous-inviscid iterations (16) are convergent.

(2) The convergence rate of the viscous-inviscid iterations decreases monotonically with the number of offdiagonals dropped.

Proof

(1) Since $E-V$ and $M \equiv I-V$ are $M$-matrices and $N \equiv I-E \geq 0$, the splitting $E-V=(I-V)-(I-E)$ is regular and convergent (Theorem A.2), i.e., $\rho\left(M^{-1} N\right)=\rho\left((I-V)^{-1}(I-E)\right)<1$, which proves convergence of the VII iterations.

(2) Next let $I_{a}$ and $I_{b}$ be two different interaction laws, where $I_{b}$ contains less off-diagonals and/or a larger main diagonal, hence $I_{b} \geq I_{a}$. Then $0 \leq N_{a} \equiv I_{a}-E \leq I_{b}-E \equiv N_{b} \geq 0$, and since the splittings of the $M$-matrix $E-V$ are regular we have (Theorem A.2)

$\rho\left(\left(I_{a}-V\right)^{-1}\left(I_{a}-E\right)\right) \leq \rho\left(\left(I_{b}-V\right)^{-1}\left(I_{b}-E\right)\right)<1$,

which proves the second part of the theorem.

\subsection{Boundary-layer iterations}

During each VII iteration a boundary-layer computation has to be performed in which (16) is solved. This is done by repeated marching through the boundary layer, starting near the stagnation point and proceeding in a downstream direction. Thus, a Gauss-Seidel type of iteration is performed:

$\left(I_{d}-I_{l}-V\right) \delta^{*^{(k)}}=I_{u} \delta^{*^{(k-1)}}$.

Here $I=I_{d}-I_{l}-I_{u}$, with $I_{d}$ a diagonal matrix, $I_{l}$ a strictly lower triangular matrix and $I_{u}$ a strictly upper triangular matrix. Further $(k)$ indicates the $k$ th boundary-layer iteration. This method 'only' has to iterate on the upper triangular part of the matrix $I-V$, which here consists of entries from $I$. Hence it may be expected that a 'small' upper-diagonal part will speed up convergence of the boundary-layer sweeps. Also, an increase of the matrix diagonal is expected to be profitable. Indeed, under Assumption 1 it can be proven that the Gauss-Seidel convergence improves monotonically with the number of dropped off-diagonals in $I$. The following theorem gives a precise statement $[42,43]$.

Theorem 2 Let the interaction law I be constructed such that I $\geq$ E. Moreover, let E and I satisfy Assumption 1. Then:

(1) The boundary-layer iterative process (17) is convergent.

(2) The convergence rate of the boundary-layer iterations increases monotonically with the number of off-diagonals dropped. 
Proof

(1) As in the proof of Theorem 1, convergence of the Gauss-Seidel iterations (17) is guaranteed because under the assumptions the corresponding splitting is regular (Theorem A.2).

(2) Next, let $I_{a}=\left(I_{d}\right)_{a}-\left(I_{l}\right)_{a}-\left(I_{u}\right)_{a}$ and $I_{b}=\left(I_{d}\right)_{b}-\left(I_{l}\right)_{b}-\left(I_{u}\right)_{b}$ be two different interaction laws, where $I_{b}$ contains less off-diagonals and/or a larger main diagonal. Hence, $\left(I_{d}\right)_{b} \geq\left(I_{d}\right)_{a},\left(I_{l}\right)_{b} \leq\left(I_{l}\right)_{a}$ and $0 \leq\left(I_{u}\right)_{b} \leq\left(I_{u}\right)_{a}$. It follows that $\left(I_{d}\right)_{b}-\left(I_{l}\right)_{b}-V \geq\left(I_{d}\right)_{a}-\left(I_{l}\right)_{a}-V$. Since $V$ does not have upper-diagonal entries, under Assumption 1 both are $M$-matrices, and Theorem A.6 yields $0 \leq\left(\left(I_{d}\right)_{b}-\left(I_{l}\right)_{b}-V\right)^{-1} \leq$ $\left(\left(I_{d}\right)_{a}-\left(I_{l}\right)_{a}-V\right)^{-1}$. Further, straightforward multiplication of the non-negative matrices involved shows that the Gauss-Seidel iteration matrices for the two interaction laws satisfy $0 \leq\left(\left(I_{d}\right)_{b}-\left(I_{l}\right)_{b}-V\right)^{-1}\left(I_{u}\right)_{b} \leq$ $\left(\left(I_{d}\right)_{a}-\left(I_{l}\right)_{a}-V\right)^{-1}\left(I_{u}\right)_{a}$. Thus the iteration matrices are non-negative, and the Perron-Frobenius theorem (Theorem A.4) states that a similar inequality holds for their spectral radii. Hence Gauss-Seidel iterations for interaction law $I_{b}$ converge at least as fast as those for $I_{a}$.

Theorems 1 and 2 state that the convergence of the boundary-layer iterations reacts opposite to the convergence of the VII iterations: the VII iterations prefer an interaction law I which resembles the 'exact' inviscid flow, whereas the boundary-layer iterations prefer a 'small' interaction law. Especially, it is remarked that an interaction law that only consists of a main diagonal does not require boundary-layer iterations at all. We will discuss this trade-off in Sect. 7.1.

\subsection{Robustness}

As already discussed in Sect. 3, the boundary-layer formulation is highly nonlinear. In Fig. 6 we copy Fig. 3, where, at a fixed boundary-layer station, the dependence between the edge velocity $u_{e}$ and the displacement thickness $\delta^{*}$ is shown [40]. For a solution to exist, in terms of Fig. 6, the slope of the inviscid-flow relation has to be sufficiently large (at least larger than the asymptotic slope of the boundary-layer relation). In more mathematical terms, the eigenvalues of $E-V$ should all lie in the stable half plane.

When an interaction law is selected, a similar reasoning applies. In particular we do not want the iterations to break down because of unfavourable properties of $I-V$. In Fig. 6, an interaction law with a smaller slope than $E$ could jeopardize the calculations; an example is indicated by "non-robust interaction law". A safer way is to choose the slope of $I$ steeper than that of $E$, as indicated by "robust interaction law". In mathematical terms, we want the eigenvalues of $I-V$ to be sufficiently far away from the imaginary axis, at least as far as those of $E-V$. The distance from the axis is given by the minimum real part of the eigenvalues; this quantity is defined as $\tau(I-V)$ (see Theorem A.5).

Again, theory can be developed. Theorem 3 shows that $\tau(I-V)>\tau(E-V)$, hence the eigenvalues of $I-V$ are further away from the imaginary axis than those of $E-V$. Moreover, the distance to the axis grows with the

Fig. 6 Boundary layer, inviscid flow and some interaction laws. The latter's slope should be sufficiently large to allow intersection with the boundary-layer relation

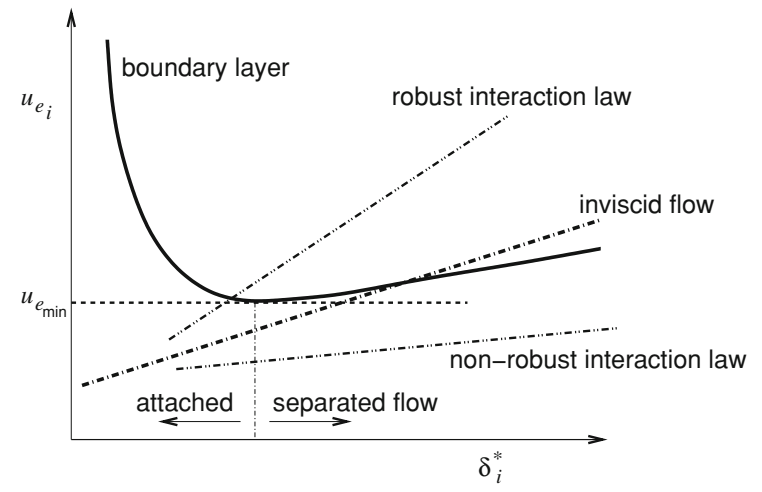


number of dropped off-diagonals, herewith increasing the robustness of the boundary-layer calculation. Also in this robustness respect, an interaction law consisting of only the main diagonal of $E$ scores best.

Theorem 3 Let the interaction law I be constructed such that I $\geq$ E. Moreover, let $E$ and I satisfy Assumption 1. Let $\tau(A)$ denote the minimum real part of the eigenvalues of $A$. Then:

(1) $\tau(I-V) \geq \tau(E-V)$;

(2) $\tau(I-V)$ is a non-decreasing function of the number of outer off-diagonals set to zero and of the magnitude of the diagonal entries, respectively.

Proof By assumption, both $E-V$ and $I-V$ are $M$-matrices; moreover $I-V \geq E-V$. Then Theorem A.5 immediately gives $\tau(I-V) \geq \tau(E-V)$, which proves the first part of the theorem. Next, let $I_{a}$ and $I_{b}$ be two different interaction laws, where $I_{b}$ contains less off-diagonals and/or a larger main diagonal. This gives $I_{b} \geq I_{a}$, hence $I_{b}-V \geq I_{a}-V$. Again, Theorem A.5 yields $\tau\left(I_{b}-V\right) \geq \tau\left(I_{a}-V\right)$, which completes the proof.

Corollary 1 Let the interaction law I be constructed from E by dropping off-diagonals or by increasing its diagonal. Then:

(1) $\tau(I) \geq \tau(E)$;

(2) $\tau(I)$ is a non-decreasing function of the number of outer off-diagonals set to zero and of the magnitude of the diagonal entries, respectively.

Proof According to its construction $I \geq E$; moreover both are $M$-matrices. Hence they satisfy Assumption 1 for the case $V \equiv 0$. Then application of Theorem 3 with $V=0$ does the rest.

In terms of Fig. 6, the drawn slopes of the inviscid flow operator $E$ and the interaction laws $I$ correspond with their smallest eigenvalue. All other eigenvalues would lead to steeper lines. A quantity like $\tau(E-V)$ corresponds with the difference in slope of the inviscid-flow relation and the boundary relation. As long as this difference is positive we may expect a solution of the nonlinear viscous-inviscid problem to exist. In this respect, Theorem 3 and its corollary guarantee that the proposed interaction laws (obtained by omitting diagonals of $E$ ) are lying on the robust side in Fig. 6, with slopes larger than that of the targeted 'exact' inviscid flow.

\section{Simplified interaction in practice}

\subsection{Indented plate}

The theory behind simplifying the interaction law will first be tested with the above indented plate (Fig. 4). As suggested by the theory, the interaction law $I$ is chosen by simply dropping off-diagonals in the 'exact' inviscid flow matrix $E$. However, there is no rigorous proof that Assumption 1 is satisfied, an assumption which was quite helpful in developing the theory. Nevertheless, let us see what happens.

A large number of calculations was performed in which the number of dropped diagonals was varied from 0 (i.e., $I=E$ ) to all but one (i.e., $I=\operatorname{diag}\left(4 U_{\infty} / \pi h\right)$ ). All calculations did converge (as predicted by Theorem 3 ), albeit at various convergence rates. Let us summarize them.

Figure 7 (left) gives the number of VII iterations as a function of the number of retained off-diagonals. Three flow situations have been distinguished: one with attached flow (when the dent is very shallow), one with mild separation, and one with severe separation (as in Fig. 4). For all choices of $I$ the VII iterations are found to converge. Moreover, in line with Theorem 1, the convergence of the VII iterations improves monotonically with the number of diagonals retained in $I$. For large numbers of off-diagonals, the limit number of iterations is $2-3$. For attached flow this can be compared with the direct method which also requires three iterations to converge (in the separated flow cases the direct method breaks down). 

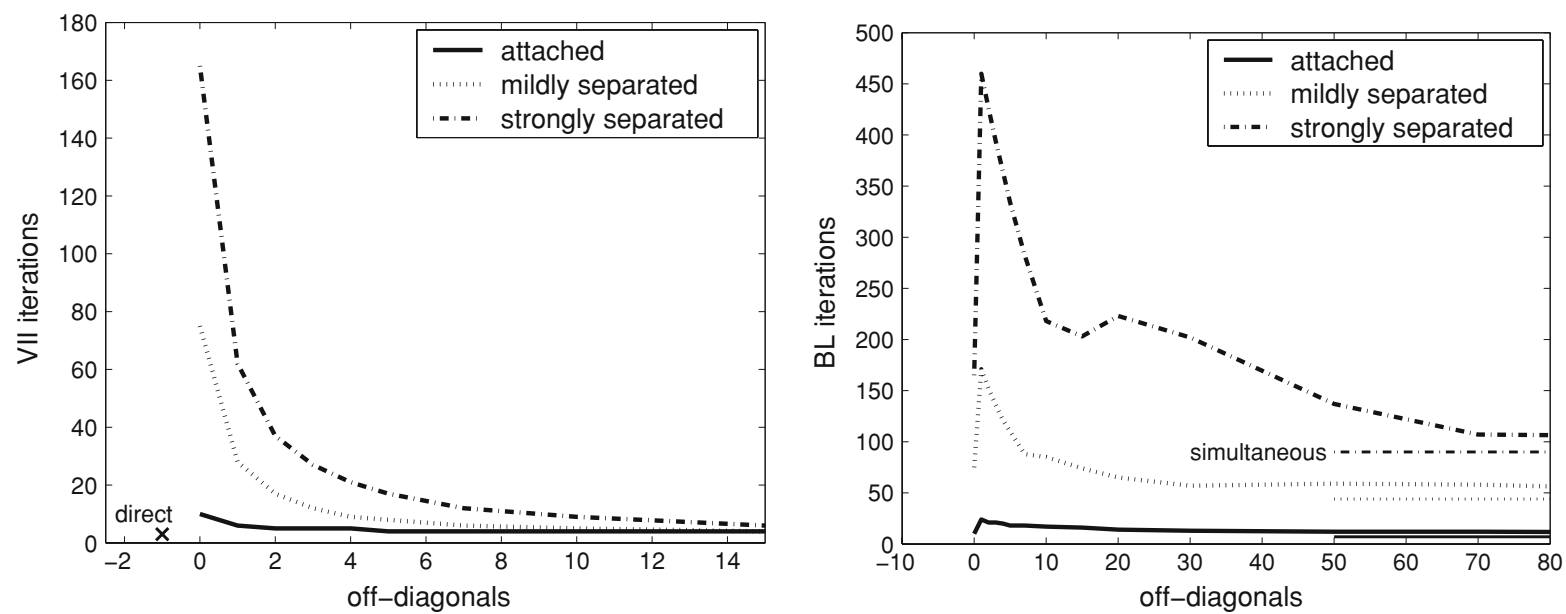

Fig. 7 Number of VII iterations (left) and total number of boundary-layer sweeps (right) as a function of the number of retained off-diagonals

When the number of retained off-diagonals is increased, the number of VII iterations drops fast. However, it leads to slower convergence of the boundary-layer iterations (in line with Theorem 2). Thus, an essential question is whether this increase in boundary-layer sweeps can be compensated by a decrease in VII iterations. Therefore the total number of boundary-layer sweeps (i.e., from all VII iterations added together) has also been monitored in Fig. 7 (right). A local minimum is found to exist when the interaction law only consists of the main diagonal (in this case one boundary-layer sweep per VII iteration suffices). When off-diagonals are added, the average number of Gauss-Seidel sweeps per VII iteration is found to increase, ${ }^{1}$ as predicted by Theorem 2 . The important observation is that, when adding more off-diagonals, the decrease in VII iterations becomes dominant: a minimum total number of boundary-layer sweeps is found in the limit $I \rightarrow E$. In this limit, the number of boundary-layer sweeps should be equal to the number required for a fully simultaneous treatment, i.e., when (15) is solved by Gauss-Seidel. This is indeed the case.

Thus for the interaction law two interesting choices exist. One option is to choose it according to the 'full' external flow; the other option is to choose it equal to only the diagonal $4 U_{\infty} / \pi h$ of the inviscid flow matrix. As the first option is against our quest for simplicity, below we will test the second option on a realistic problem of boundary-layer flow past a two-dimensional airfoil.

\subsection{Subsonic airfoil flow}

The above ideas on simplifying the interaction law will now be tested for aerodynamic flow past a NACA 0012 airfoil (at $\operatorname{Re}=9 \times 10^{6}$, and $M_{\infty}=0$ ); experimental data is available, e.g. [48, App. IV]. The inviscid flow is modelled by potential theory, and computed by means of a panel method [42]. The boundary layer is modelled with the steady version of Head's entrainment method (2). It is solved together with the diagonal interaction law

$I=\operatorname{diag}\left\{4 U_{\infty} / \pi h\right\}$.

We stress that this interaction law is unaware of the Kutta condition and the latter's effect on the global circulation; it only accounts for the local VII physics-but this turns out to be sufficient. Surely, the Kutta condition is part of the 'exact' inviscid flow as denoted by the inviscid flow operator $E$ in (12). Its influence on the global flow pattern is accounted for through the VII iterations (13).

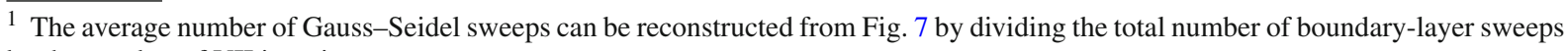
by the number of VII iterations. 
Fig. 8 Lift polar for NACA0012 airfoil: viscous-inviscid calculation versus experiment

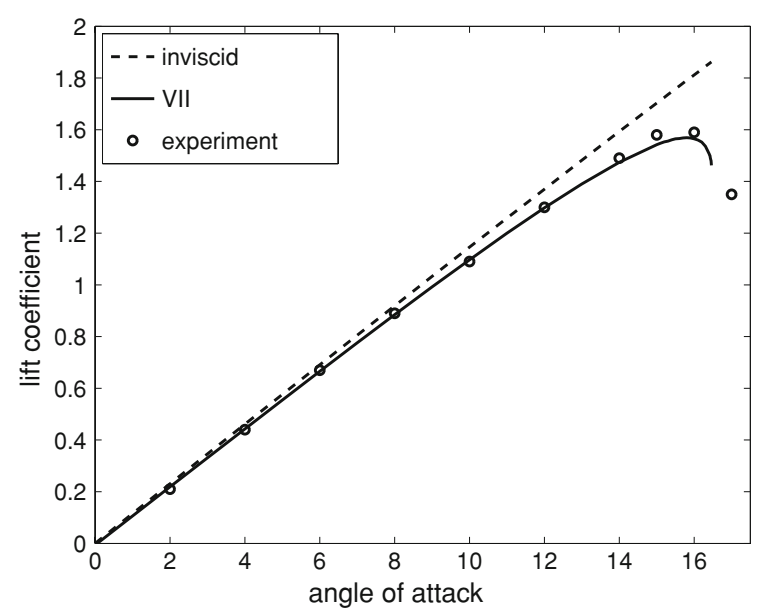

A large part of the lift polar has been computed (Fig. 8). The calculations turn out to be highly robust. It appears that even for separated-flow cases beyond maximum lift, the calculations converge without any need for a good initial guess; they can be started from scratch! The number of VII iterations with the extremely simple interaction law (18) typically is less than 100 at zero lift up to 1000 around maximum lift. Only for larger angles of attack, beyond maximum lift when stall is setting in, the computations break down. This robust numerical behaviour is all due to the selected interaction law. The number of iterations may sound large, but they can easily can be combined with the time-stepping in an unsteady inviscid flow solver. Anyway, for two-dimensional simulations, the computing times count in just seconds on an average PC.

Finally, the agreement of the computational results with experiment is quite good. However, here the interaction law deserves no credit; the latter is merely taking care that converged results are obtained. The integral method described in Sect. 2 is doing the fine job here, although it was basically developed in the 1970s. Navier-Stokes solutions still have a hard time to yield similar results [1,2].

\section{Epilogue}

Prandtl's 1904 boundary-layer theory formed the starting point for the viscous-inviscid interaction methods that have been developed in the last two decades of the 20th century. They have become very popular, since in comparison with brute-force Navier-Stokes solutions they are about two orders less expensive, whereas for flow conditions with thin shear layers the results are equally useful. Because of their modest computational complexity, they are ideal to be used in aerodynamic optimization studies [15,49,50] or in multi-physics problems like aeroelasticity [51].

The greatest challenge has been to understand and resolve the singularity at separation, which occurs when the boundary-layer equations are solved with prescribed pressure. In 1948, Goldstein [4] already foresaw the possibility that near separation in general no solution does exist, unless the pressure satisfies certain properties.

In the 1950s Lighthill wrote some further seminal papers on the subject [5,32], with the role of hierarchy already visible between the lines. His research inspired Stewartson and contemporaries to develop the asymptotic framework valid near separation: the triple deck [41]. In 1975, Lagerstrom [12] described his view on the triple deck: boundary layer and inviscid flow have to be solved simultaneously. A few years later, LeBalleur [27] and Veldman [17,39] showed in engineering terms where the problems at separation came from. It is through this type of insight that the use of viscous-inviscid interaction methods in engineering applications could flourish.

The quasi-simultaneous method is one of the methods that emerged. It makes use of an interaction law to be solved simultaneously with the boundary-layer equations. In the paper, mathematical ideas and arguments have been presented to select such an interaction law. Aspects like convergence rate and robustness have been discussed. Again, one-century old science plays an important role: the theory of Perron and Frobenius on non-negative matrices. 
An interesting question, also of high practical relevance, is how close one can get to Prandtl's original boundarylayer formulation of prescribed edge velocity without running into Goldstein's singularity. It was found that a simple modification suffices

$$
\left(u_{e}-\frac{4 U_{\infty}}{\pi h} \delta^{*}\right)^{\text {(new) }}=\left(u_{e}-\frac{4 U_{\infty}}{\pi h} \delta^{*}\right)^{\text {(old) }} .
$$

This slight change, unlikely to be further simplified, results in a highly robust calculation method for separated flow, even applicable to airfoil calculations beyond maximum lift. I am quite sure that Sir James Lighthill would have liked the simplicity of this approach, as well as the combined mathematical and physical reasoning behind it [52].

Acknowledgements The author would like to acknowledge the enthusiastic and highly valuable contributions from his PhD students Edith Coenen and Henny Bijleveld.

Open Access This article is distributed under the terms of the Creative Commons Attribution Noncommercial License which permits any noncommercial use, distribution, and reproduction in any medium, provided the original author(s) and source are credited.

\section{Appendix: Matrix prerequisites}

In the above analysis use is made of a number of well-known results on matrices and iterative methods, which are collected here. A dominant role is played by $M$-matrices defined as [44, Chap. 3.5; 45, Chap. 2.7; 46, Chap. 2.5]:

Definition A.1 ( $M$-matrix) A real matrix $A=\left(a_{i, j}\right)$ with $a_{i, j} \leq 0$ for all $i \neq j$ is an $M$-matrix if $A$ is nonsingular, and $A^{-1} \geq 0$.

Especially, the latter property is very helpful in producing regular splittings $A=M-N$, with $M^{-1} \geq 0, N \geq 0$, for which $M x^{(k+1)}=N x^{(k)}+b$ converges.

Theorem A.2 (Convergent splittings for $M$-matrices) When A, $M_{1}$ and $M_{2}$ are $M$-matrices, whereas $0 \leq N_{1} \leq N_{2}$, then the splittings $A=M_{i}-N_{i}(i=1,2)$ are regular and convergent. In particular we have $0 \leq \rho\left(M_{1}^{-1} N_{1}\right) \leq$ $\rho\left(M_{2}^{-1} N_{2}\right)<1$.

Proof The proof follows e.g. from Theorem 3.13 and Theorem 3.15 in Varga [44, Chap. 3.6] and/or Theorem 5.5 and Corr. 5.6 in Young [45, Chap. 4].

Diagonal dominance, in combination with the correct sign pattern, produces $M$-matrices, as formulated in the next theorem from Varga [44, p. 85]:

Theorem A.3 (Diagonal dominance) If $A=\left(a_{i, j}\right)$ is a real, irreducibly diagonally dominant matrix with $a_{i, j} \leq 0$ for all $i \neq j$, and $a_{i, i}>0$ for all $i$, then $A^{-1}>0$. Hence $A$ is an irreducible $M$-matrix.

$M$-matrices are closely related to non-negative matrices. For the latter we have the theorem of Perron (1907) and Frobenius (1912), which makes statements about their largest eigenvalue. For $M$-matrices this leads to information on their eigenvalues with smallest real part; see especially Horn and Johnson [46, pp. 128-131].

Theorem A.4 (Perron-Frobenius) Let $A \geq 0$ be an irreducible matrix. Then:

(1) A has a positive real eigenvalue equal to its spectral radius.

(2) $\rho(A)$ increases when any entry of A increases.

Proof Varga [44, Theorem. 2.1].

Theorem A.5 (Minimum eigenvalue of $M$-matrices) Let $A$ be an irreducible $M$-matrix, and let $\tau(A)$ be defined as the minimum real part of its eigenvalues, i.e., $\tau(A)=\min _{\lambda} \mathfrak{R e}(\lambda(A))$. Then: 
(1) A has a real eigenvalue equal to $\tau(A)$.

(2) $\tau(A)$ decreases when any entry of A decreases.

Proof For sufficiently large $\alpha>0$ we can write $A=\alpha I-\hat{A}$, where $\hat{A}$ is a non-negative matrix. Next apply the Perron-Frobenius theorem to $\hat{A}$.

Finally, we need a comparison theorem on inverses of $M$-matrices:

Theorem A.6 (Inverse comparison) Let $A$ and $B$ be $M$-matrices, with $A \leq B$. Then $A^{-1} \geq B^{-1}$.

Proof Horn and Johnson [46, p. 117].

\section{References}

1. Holst TL (1987) Viscous transonic airfoil workshop - Compendium of results. In: AIAA paper 87-1460, Honolulu, HI

2. Cebeci T (1999) An engineering approach to the calculation of aerodynamic flows. Springer, Berlin

3. Prandtl L (1905) Ueber Fluessigkeitsbewegung mit kleiner Reibung. In: Verhandlungen des dritten internationalen Mathematischen Kongresses, Heidelberg. Teubner Verlag, Leipzig, pp 484-491

4. Goldstein S (1948) On laminar boundary layer flow near a point of separation. Quart J Mech Appl Math 1:43-69

5. Lighthill MJ (1953) On boundary layers and upstream influence I.A comparison between subsonic and supersonic flows. Proc Roy Soc Lond A 217:478-507

6. Lighthill MJ (2000) Upstream influence in boundary layers 45 years ago. Philos Trans Roy Soc Lond A 358:3047-3061

7. Hayes WD, Probstein RF (1959) Hypersonic flow theory. Academic Press, New York

8. Catherall D, Mangler KW (1966) The integration of the two-dimensional laminar boundary-layer equations past the point of vanishing skin friction. J Fluid Mech 26:163-182

9. Stewartson K (1969) On the flow near the trailing edge of a flat plate II. Mathematika 16:106-121

10. Neiland VYa (1969) Towards a theory of separation of the laminar boundary layer in a supersonic stream. Mekh Zhid Gaza 4:53-57 [English transl. (1970) Fluid Dyn 4:33-55]

11. Messiter AF (1970) Boundary-layer flow near the trailing edge of a flat plate. SIAM J Appl Math 18:241-257

12. Lagerstrom PA (1975) Solutions of the Navier-Stokes equation at large Reynolds number. SIAM J Appl Math 28:202-214

13. LeBalleur JC (1978) Couplage visqueux-non visqueux: méthode numérique et applications aux écoulements bidimensionnels transsoniques et supersoniques. La Recherche Aérospatiale 183:65-76

14. Carter JE (1981) Viscous-inviscid interaction analysis of turbulent separated flow. In: AIAA paper 81-1241, Palo Alto, CA

15. Drela M, Giles MB (1987) ISES: a two-dimensional viscous aerodynamic design and analysis code. In: AIAA paper $87-1118$

16. Moses HL, Jones RR III, O'Brien WF Jr, Peterson RP (1978) Simultaneous solution of the boundary layer and freestream with separated flow. AIAA J 16:61-66

17. Veldman AEP (1979) A numerical method for the calculation of laminar incompressible bounday layers with strong viscous-inviscid interaction. Report NLR TR 79023, National Aerospace Laboratory, Amsterdam

18. Veldman AEP (1981) New, quasi-simultaneous method to calculate interacting boundary layers. AIAA J 19:79-85

19. Veldman AEP, Lindhout JPF, de Boer E, Somers MAM (1990) Vistrafs: a simulation method for strongly-interacting viscous transonic flow. In: Cebeci T (ed) Numerical and physical aspects of aerodynamic flow IV. Springer, Berlin, pp 37-51

20. Veldman AEP (2001) Matched asymptotic expansions and the numerical treatment of viscous-inviscid interaction. J Eng Math 39:189-206

21. Veldman AEP (2005) Quasi-simultaneous viscous-inviscid interaction for transonic airfoil flow. In: AIAA paper 2005-4801

22. Cebeci T, Cousteix J (1999) Modelling and computation of boundary-layer flows. Springer, Berlin

23. Smith FT (1991) Steady and unsteady 3-D interactive boundary layers. Comput Fluids 20:243-268

24. Schlichting H, Gersten K (2000) Boundary-layer theory, 8th rev. edn. Springer, Berlin

25. Lock RC, Williams BR (1987) Viscous-inviscid interactions in external aerodynamics. Prog Aerosp Sci 24:51-171

26. Head MR (1960) Entrainment in the turbulent boundary layer. Aeronautical Research Council, Reports and Memoranda 3152

27. LeBalleur JC (1977) Couplage visqueux-non visqueux: analyse du problème incluant décollements et ondes de choc. La Recherche Aérospatiale 1977-6:349-358

28. Lock RC (1985) Prediction of the drag of wings at subsonic speeds by viscous-inviscid interaction techniques. In: AGARD-R-723, paper 10

29. Houwink R, Veldman AEP (1984) Steady and unsteady separated flow computation for transonic airfoils. In: AIAA paper 84-1618

30. Reynolds WC, Cebeci T (1976) Calculation of turbulent flows. In: Bradshaw P (ed) Turbulence. Topics in applied physics, vol 12. Springer, Berlin, pp 193-229

31. Sandham ND (2008) Transitional separation bubbles and unsteady aspects of aerodynamic stall. Aeronaut J 112:395-404

32. Lighthill MJ (1958) On displacement thickness. J Fluid Mech 4:383-392 
33. Cousteix J (1986) Three-dimensional and unsteady boundary-layer computations. Annu Rev Fluid Mech 18:173-196

34. Van Dommelen LL, Shen SF (1980) The spontaneous generation of the singularity in a separating laminar boundary layer. J Comput Phys 38:125-140

35. Henkes RAWM, Veldman AEP (1987) On the breakdown of the steady and unsteady interacting boundary-layer description. J Fluid Mech 179:513-529

36. Stewartson K, Smith FT, Kaups K (1982) Marginal separation. Stud Appl Math 67:45-61

37. Smith FT (1986) Steady and unsteady boundary-layer separation. Annu Rev Fluid Mech 18:197-220

38. Elliott JW, Smith FT (1987) Dynamic stall due to unsteady marginal separation. J Fluid Mech 179:489-512

39. Veldman AEP (1980) Boundary layers with strong interaction: from asymptotic theory to calculation method. In: Miller JJH (ed) Proceedings of the BAIL 1 conference on boundary and interior layers. Boole Press, Dublin, pp 149-163

40. Veldman AEP (1984) A numerical view on strong viscous-inviscid interaction. In: Habashi WG (ed) Computational methods in viscous flows. Pineridge Press, Southampton, pp 343-363

41. Stewartson K (1974) Multi-structured boundary layers on flat plates and related bodies. Adv Appl Mech 14:145-239

42. Coenen EGM (2001) Viscous-inviscid interaction with the quasi-simultaneous method for 2D and 3D airfoil flow. PhD thesis, University of Groningen. www.ub.rug.nl/eldoc/dis/science/e.g.m.coenen

43. Coenen EGM (1999) Quasi-simultaneous coupling for wing and airfoil flow. In: Lai C-H, Bjorstad CE, Cross M, Widlund OB (eds) Domain decomposition methods in science and engineering. Domain Decomposition Press, Bergen, pp 197-205

44. Varga RS (1962) Matrix Iterative Analysis. Prentice-Hall, Englewood Cliffs

45. Young DM (1971) Iterative solution of large linear systems. Academic Press, London

46. Horn RA, Johnson CR (1991) Topics in matrix analysis. Cambridge University Press, Cambridge

47. Carter JE, Wornom SF (1975) Solutions for incompressible separated boundary layers including viscous-inviscid interaction. In: Aerodynamic analysis requiring advanced computers, NASA SP-347, pp 125-150

48. Abbott IH, Von Doenhoff AE (1959) Theory of wing sections. Dover, New York

49. Smith PD (1989) A viscous package for attached and separated flows on swept and tapered wings. RAE Technical Report 89027

50. Van der Wees AJ, van Muijden J (1993) A fast and robust viscous-inviscid interaction solver for transonic flow about wing/body configurations on the basis of full potential theory. In: AIAA paper 93-3026

51. Edwards JW (2008) Calculated viscous and scale effects on transonic aeroelasticity. J Aircr 45:1863-1871

52. Lighthill MJ (1971) The art of teaching the art of applying mathematics. Math Gaz 55:249-270 